\title{
LIVER
}

\section{Effect of antiviral treatment on evolution of liver steatosis in patients with chronic hepatitis $C$ : indirect evidence of a role of hepatitis $C$ virus genotype 3 in steatosis}

\author{
L Castéra, C Hézode, F Roudot-Thoraval, I Lonjon, E-S Zafrani, J-M Pawlotsky, D Dhumeaux
}

Gut 2004;53:420-424. doi: 10.1136/gut.2003.009936

See end of article for authors' affiliations

Correspondence to

Dr L Castera, Service

d'Hépato-gastrentérologie,

Hôpital Haut Lévêque,

Avenue Magellan, 33604

Pessac, France;

laurent.castera@

chu-bordeaux.fr

Accepted for publication 11 July 2003

\begin{abstract}
Background and aim: Recent studies suggest that liver steatosis in chronic hepatitis $C$ may be the expression of a direct cytopathic effect of hepatitis $\mathrm{C}$ virus (HCV), particularly in patients infected with genotype 3. To investigate this hypothesis, we studied the relationship between steatosis evolution and $\mathrm{HCV}$ clearance after antiviral treatment in patients with chronic hepatitis $\mathrm{C}$ and paired liver biopsies.

Methods: A total of 151 patients (37 with HCV genotype 3; 114 with HCV non-3 genotypes) were selected according to the following criteria: presence of steatosis at initial biopsy; no antiviral treatment prior to the first biopsy; antiviral treatment received between the two biopsies; body mass index (BMI) $<28 \mathrm{~kg} / \mathrm{m}^{2}$; absence of excessive alcohol intake; no serum hepatitis B surface antigen or human immunodeficiency virus antibodies; and absence of diabetes mellitus. Evolution of steatosis was examined by comparing steatosis grades between the two biopsies.

Results: Twenty five patients (16.5\%) were sustained virological responders (SVR) to antiviral treatment. Steatosis evolution after antiviral treatment was as follows: improvement in $36 \%$ of cases; stability in $51 \%$; and worsening in 13\%. Steatosis improvement was significantly more frequent in SVR than in nonresponders (NR) $(64 \% \vee 31 \% ; p<0.004)$. This significant difference occurred in patients infected with genotype 3 (91\% v 19\%; $<<0.0001$ ) but not in those infected with non-3 genotypes ( $43 \%$ v 34\%; NS). Among the 25 SVR, improvement in steatosis was significantly more frequent in patients infected with genotype 3 than in those infected with non-3 genotypes $(91 \% v 43 \% ; p<0.04)$ whereas in NR, improvement in steatosis did not differ between those infected with genotype 3 and non-3 genotypes (19\% v 34\%; NS). In multivariate analysis, four factors were independently associated with steatosis improvement: sustained virological response to antiviral therapy (odds ratio (OR) 6.06 (95\% confidence interval (Cl) 1.61-22.9); $p=0.01$ ), severe steatosis (OR 5.50 (95\% Cl 1.54-19.6); $p=0.01)$, HCV genotype 3 (OR 2.90 (95\% Cl 0.85-10.0); $p=0.07$ ), and $\mathrm{BMI}>25 \mathrm{~kg} / \mathrm{m}^{2}$ (OR 0.24 (95\% Cl 0.08-0.73); $\mathrm{p}=0.02)$.

Conclusions: Our results showed significant improvement in steatosis in patients infected with HCV genotype 3, who achieved sustained viral clearance. This provides further evidence for direct involvement of HCV genotype 3 in the pathogenesis of hepatic steatosis.
\end{abstract}

iver steatosis is a frequent histological finding in patients with chronic hepatitis C, occurring in more than $50 \%$ of - cases. ${ }^{1}$ This observation is of clinical relevance from both a diagnostic and prognostic point of view. Indeed, there is increasing evidence that steatosis is associated with fibrosis progression in patients with chronic hepatitis $\mathrm{C}^{2-7}$

The reason for the high frequency of steatosis in chronic hepatitis C remains poorly understood. Even when the most common causes of steatosis are carefully excluded, a significant proportion of patients with chronic hepatitis C still have steatosis. ${ }^{8}$ Since the first report of the association of steatosis with hepatitis C virus (HCV) genotype $3,{ }^{9}$ several groups, including our own, have confirmed this finding, ${ }^{24571011}$ suggesting a possible role of HCV itself in the pathogenesis of steatosis. In support of this hypothesis, other lines of evidence have come from observations of a close relationship between intrahepatic HCV RNA and the occurrence of steatosis ${ }^{4}{ }^{12}$ and the development of hepatic steatosis in transgenic mouse models expressing the HCV core protein, ${ }^{13}$ as well as structural or non-structural proteins. ${ }^{14}$ Conversely, given the high prevalence of steatosis in the general population, ${ }^{15}$ the role of host factors such as obesity, diabetes mellitus, and alcohol intake is also likely. ${ }^{16}$
Although two cases of steatosis disappearance after HCV eradication $^{4}$ and one case of steatosis recurrence after relapse of HCV infection ${ }^{17}$ have been reported in patients with genotype 3 infection, evolution of steatosis after antiviral therapy has not been rigorously investigated. In a recent study involving a relatively small number of patients, it was suggested that viral clearance after antiviral therapy could be associated with disappearance of steatosis from the liver, at least in patients with no other risk factor than HCV. ${ }^{18}$ We addressed the hypothesis that hepatic steatosis is related to HCV replication in patients with chronic hepatitis C, particularly those infected with $\mathrm{HCV}$ genotype 3. To investigate this, we studied the effect of antiviral therapy on steatosis in relation to sustained viral clearance in a series of 151 patients with chronic hepatitis $\mathrm{C}$ and paired liver biopsies.

Abbreviations: $\mathrm{HCV}$, hepatitis $\mathrm{C}$ virus; SVR, sustained virological responders; NR, non-responders; BMI, body mass index; ALT, alanine aminotransferase; $M U$, million units; $O R$, odds ratio 


\section{PATIENTS AND METHODS Study population}

Between March 1996 and October 2000, 660 consecutive patients with HCV infection (defined by positive serum antibodies to HCV by means of a second or third generation HCV enzyme linked immunosorbent assay (Ortho Diagnostic, Raritan, New Jersey, USA) and detectable serum HCV RNA (Amplicor HCV; Roche Molecular Systems, Pleasanton, California, USA) had a liver biopsy performed in our department.

A total of 151 of these patients were selected for study of steatosis evolution under antiviral therapy on the basis of: ( 1 ) the presence of steatosis (involving more than $10 \%$ of hepatocytes) at initial biopsy; (2) no antiviral treatment prior to the first biopsy; (3) at least two liver biopsies available; (4) antiviral treatment received between the two biopsies (that is, interferon-alpha or peginterferon-alpha with or without ribavirin); (5) absence of serum hepatitis B surface antigen or human immunodeficiency virus antibodies; (6) body mass index (BMI) $<28 \mathrm{~kg} / \mathrm{m}^{2}$ ); (7) absence of excessive alcohol intake $(<50 \mathrm{~g} /$ day $)$ during the six months prior to the first biopsy ${ }^{19}$; and (8) no history of diabetes mellitus.

The following epidemiological data were obtained from all patients: age at diagnosis, sex, source of infection (transfusion, intravenous drug use, other source, unknown source), age at exposure and duration of infection in patients with a known risk factor (calculated as the interval between the presumed date of infection and date of diagnosis), interval between the two biopsies, and time from the end of antiviral treatment to the post-treatment biopsy.

\section{Definition of the response to antiviral therapy}

Patients with both normal alanine aminotransferase (ALT) levels and undetectable serum HCV RNA six months after interferon withdrawal were considered sustained virological responders (SVR). The remaining patients were considered non-responders (NR).

\section{Virological tests}

Serum antibodies to HCV were detected by means of a second or third generation HCV enzyme linked immunosorbent assay (Ortho Diagnostic), according to the manufacturer's instructions. All of these tests were routinely performed at the time of the first visit to our unit.

HCV genotype was determined in all patients using a line probe assay (Inno-LiPA HCV II; Innogenetics, Ghent, Belgium). Genotypes were classified according to Simmonds and colleagues. ${ }^{20}$

HCV RNA was determined in all patients using a commercial assay (Amplicor HCV; Roche Molecular Systems) at the time of the first biopsy, before initiation of antiviral treatment, and six months after treatment discontinuation.

HCV viral load was determined by means of the second generation branched DNA assay (Quantiplex HCV RNA 2.0; Bayer Diagnostics, Emeryville, California, USA) on stored serum samples at the time of the first biopsy.

\section{Histological studies and steatosis evaluation}

Liver biopsy specimens of more than $10 \mathrm{~mm}$ in length were fixed in formalin, paraffin embedded, and stained with haematoxylin-eosin-safran, or picroSirius red for collagen and Perls' technique for iron. Paired biopsy samples were read by the same pathologist (ESZ) who was unaware of patient demographic, clinical, and virological data or of the timing and sequence of the biopsy specimens.

Steatosis was graded on the two liver biopsies according to the Metavir classification system, ${ }^{21}{ }^{22}$ as previously validated: ${ }^{7}$ none; mild (involving less than $10 \%$ of hepatocytes); moderate (involving 10-30\% of hepatocytes); and severe (involving more than $30 \%$ of hepatocytes). Evolution of steatosis was assessed by comparing the grades between the two biopsies: improvement was defined as a decrease of at least one grade; worsening was defined as an increase of at least one grade; and stability was defined as identical steatosis grades.

\section{Statistical analysis}

Descriptive statistics are shown as means (SD). One way analysis of variance (ANOVA) or the Mann-Whitney test was used when necessary for statistical comparison of quantitative data. The $\chi^{2}$ test or Fisher's exact test was used for qualitative data. A p value $<0.05$ was considered significant. Given the small size of the sample, comparisons were between patients with improvement of steatosis and those with stability or worsening of steatosis.

To assess the independent value of each parameter related to steatosis improvement in the univariate analysis $(p<0.1)$, a multivariate analysis was performed by means of a stepwise logistic regression analysis. All analyses were performed using BMDP statistical software (BMDP statistical software Inc, Los Angeles, California, USA).

\section{RESULTS}

\section{General characteristics of patients at the time of the first biopsy}

Patient characteristics are shown in table l. They were 111 males and 40 females, with a mean age of 42.9 (11.4) years. Routes of transmission were blood transfusion in $35.1 \%$ of cases, intravenous drug use in $26.5 \%$, and other or unknown in $38.4 \%$. Thirty seven patients $(24.5 \%)$ were infected with genotype 3 whereas $59.6 \%$ were infected with genotype 1 , $11.3 \%$ with genotype 2 , and $4.6 \%$ with genotype 4 . According to our classification, steatosis was mild in $55.0 \%$ of cases, moderate in $21.2 \%$, and severe in $23.8 \%$. Severe steatosis was significantly associated with HCV genotype $3(\mathrm{p}=0.0003)$. Nine patients (eight with non-3 genotypes) had evidence of more severe histological lesions of steatohepatitis.

Most patients were treated in therapeutic trials with different regimens: 125 were treated for 6-12 months with interferon-alpha alone (3-6 million units (MU) three times per week), 23 were treated for 6-12 months with interferonalpha (3-6 MU thrice per week) plus ribavirin (1000$1200 \mathrm{mg} /$ day according to body weight), and three were treated for 6-12 months with peginterferon-alpha ( $1.5 \mu \mathrm{g} / \mathrm{kg}$ once weekly) and ribavirin (1000-1200 mg/day according to body weight). The cumulative dose of interferon-alpha received was 325 (177) MU (range 108-820) with a mean duration of 7.6 (3.1) months (range 3-12). Dose and duration of interferon-alpha treatment did not differ between patients infected with genotype 3 and those infected with non-3 genotypes (table 1). Overall, 25 patients (16.5\%) achieved sustained viral clearance. Eleven were infected with genotype 3 whereas 10 were infected with genotype 1 and four with genotype 2 .

\section{Relationship between steatosis evolution and viral clearance after antiviral therapy}

The mean interval between the two biopsies was 24.6 (10.4) months and the mean time from the end of antiviral treatment to the post-treatment biopsy was 12.3 (8.7) months. This did not differ between SVR and NR patients (9.6 (3.2) $v 13.2(9.2)$, respectively; NS), or between patients infected with genotype 3 and those infected with non-3 genotypes (12.9 (11.5) v 12.2 (7.6), respectively; NS).

Steatosis evolution after antiviral treatment was as follows: improvement in $36 \%$ of cases, stability in $51 \%$, and worsening 
Table 1 Characteristics of the 151 patients at the time of the first biopsy, according to hepatitis $\mathrm{C}$ virus (HCV) genotype

\begin{tabular}{|c|c|c|c|c|}
\hline & \multicolumn{4}{|l|}{ HCV genotype } \\
\hline & Total $(n=151)$ & $3(n=37)$ & Non-3 $(n=114)$ & $\mathrm{p}$ Value \\
\hline Age (y) & $42.9(11.4)$ & $34.4(5.3)$ & $45.7(11.7)$ & $<0.0001^{*}$ \\
\hline $\operatorname{Sex}(M / F)$ & $111 / 40$ & $31 / 6$ & $80 / 34$ & NS \\
\hline \multicolumn{5}{|l|}{ Source of infection (\%) } \\
\hline Transfusion & 35.1 & 16 & 41 & \\
\hline IVDU & 26.5 & 65 & 14 & $<0.0001^{*}$ \\
\hline Other or unknown & 38.4 & 19 & 45 & \\
\hline Body mass index $\left(\mathrm{kg} / \mathrm{m}^{2}\right)$ & $\begin{array}{l}23.8(2.5) \\
(18.5-27.8)\end{array}$ & $22.9(2.3)$ & $23.9(2.3)$ & NS \\
\hline Body mass index $>25 \mathrm{~kg} / \mathrm{m}^{2}(\%)$ & 30.1 & 18.1 & 32.9 & NS \\
\hline Duration of infection $(y)(n=113)$ & $14.6(8.8)$ & $12.1(4.2)$ & $15.6(9.9)$ & $0.05^{\star}$ \\
\hline Age at exposure $(y)(n=113)$ & $28.0(11.5)$ & $21.7(4.2)$ & $31.2(13.0)$ & $0.0004^{*}$ \\
\hline ALT levels ( $<<5$ IU/I) & $136(90)$ & $118(63)$ & $143(95)$ & NS \\
\hline \multicolumn{5}{|l|}{ HCV genotype (\%) } \\
\hline 1 & 59.6 & & & \\
\hline 2 & 11.3 & & & \\
\hline 3 & 24.5 & & & \\
\hline 4 and other & 4.6 & & & \\
\hline HCV RNA load (mEq/ml) & $7.0(8.0)$ & $9.3(9.7)$ & $6.1(7.7)$ & NS \\
\hline \multicolumn{5}{|l|}{ Histological lesions (Metavir) } \\
\hline \multicolumn{5}{|l|}{ Activity (\%) } \\
\hline A0-1 & 29.1 & 22 & 32 & \\
\hline A2 & 64.9 & 70 & 63 & NS \\
\hline A3 & 6.0 & 8 & 5 & \\
\hline \multicolumn{5}{|l|}{ Fibrosis (\%) } \\
\hline FO-1 & 55.0 & 49 & 57 & \\
\hline F2 & 27.1 & 30 & 26 & NS \\
\hline F3-F4 & 17.9 & 21 & 17 & \\
\hline \multicolumn{5}{|l|}{ Steatosis (\%) } \\
\hline Mild (<10\% hepatocytes) & 55.0 & 24 & 65 & \\
\hline Moderate (10-30\% hepatocytes) & 21.2 & 27 & 19 & $<0.0001^{*}$ \\
\hline Severe ( $\geqslant 30 \%$ hepatocytes) & 23.8 & 49 & 16 & \\
\hline \multicolumn{5}{|l|}{ Interferon-alpha therapy } \\
\hline Cumulative dose received (MU) & $\begin{array}{l}325(177) \\
(108-820)\end{array}$ & $340(152)$ & $288(162)$ & NS \\
\hline Duration (months) & $7.6(3.1)(3-12)$ & $7.6(0.7)$ & $6.7(2.9)$ & NS \\
\hline
\end{tabular}

in $13 \%$. Steatosis improvement was significantly more frequent in SVR than in NR $(64 \% v 31 \%$; $<0.004)$ (fig 1$)$. Among SVR, mean pretreatment BMI did not differ between patients in whom steatosis improved or disappeared and those in whom it did not (23.9 (3.0) $v 24.2(2.7) \mathrm{kg} / \mathrm{m}^{2}$, respectively; NS). In addition, mean post-treatment BMI did not differ from mean pretreatment BMI (23.8 (2.7) $v 24.2$

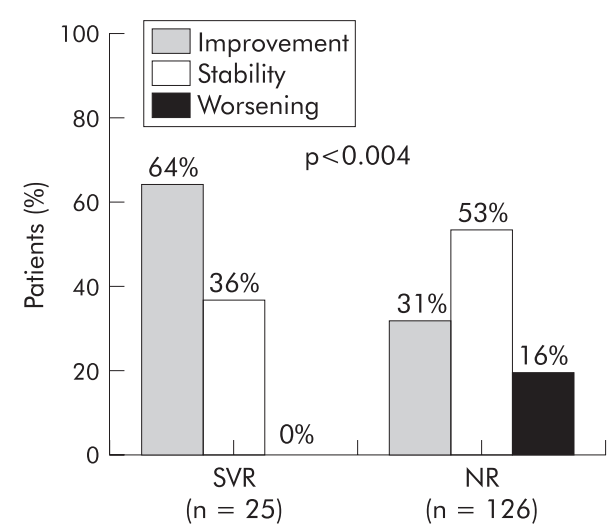

Figure 1 Relationship between steatosis evolution and sustained virological response to antiviral therapy in the 151 patients studied. Improvement was defined as a decrease of at least one grade between the two biopsies; stability was defined as identical grades between the two biopsies; and worsening of steatosis was defined as an increase of at least one grade between the two biopsies. SVR, sustained virological responders; NR, non responders.
(3.0) $\mathrm{kg} / \mathrm{m}^{2}$, respectively; NS). Similarly, although posttreatment alcohol intake was lower than before treatment (9.4 (11.2) v 10.8 (12.9) g/day, respectively), the difference did not reach statistical significance.

\section{Relationship between steatosis evolution and viral clearance according to HCV genotype}

In patients infected with HCV genotype 3, almost all SVR improved their steatosis, and steatosis improvement was significantly more frequent in patients who achieved a sustained virological response than in those who did not (91\% v 19\%; p<0.0001) (fig 2). Conversely, in patients infected with non-3 genotypes, improvement in steatosis did not differ between patients who achieved a sustained virological response and those who did not (43\% v 34\%; NS) (fig 2). Among the 25 patients who achieved a sustained virological response, improvement in steatosis was significantly more frequent in genotype 3 than in non-3 genotypes $(p<0.04)$. In patients who did not clear HCV, improvement in steatosis did not differ according to the HCV genotype.

\section{Factors independently associated with steatosis improvement}

As shown in table 2, four factors were independently associated with steatosis improvement in the multivariate analysis: a sustained virological response to antiviral therapy (odds ratio (OR) 6.06 (95\% confidence interval (CI) 1.61$22.9) ; \mathrm{p}=0.01$ ), severe steatosis at initial biopsy (OR 5.50 (95\% CI 1.54-19.6); $\mathrm{p}=0.01$ ), HCV genotype 3 (OR $2.90(95 \%$ CI $0.85-10.0) ; \mathrm{p}=0.07$ ), and $\mathrm{BMI}>25 \mathrm{~kg} / \mathrm{m}^{2}$ (OR $0.24(95 \%$ CI $0.08-0.73$ ); $\mathrm{p}=0.02$ ). 


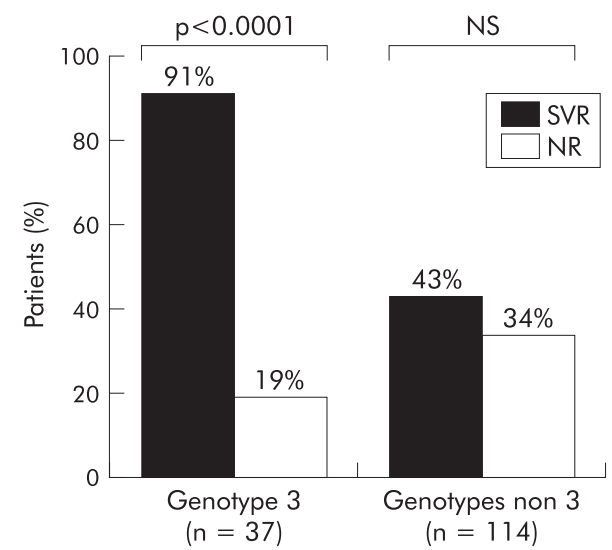

Figure 2 Relationship between steatosis improvement and sustained virological response to antiviral therapy according to genotype. Improvement was defined as a decrease of at least one grade between the two biopsies. Sustained virological responders (SVR) and nonresponders (NR) are shown. The relationship persisted after adjustment for genotype ( $p=0.004$ by Mantel-Haenszel test).

\section{DISCUSSION}

The pathogenesis of steatosis in chronic hepatitis $\mathrm{C}$ is confused by the probable existence of two distinct entities: (a) steatosis secondary to common causes such as obesity, diabetes mellitus, or alcohol intake, and (b) steatosis without known risk factors, presumably in part related to HCV. The results of the present study, which was conducted in this latter subset of patients, show a significant relationship between steatosis evolution and viral clearance after antiviral therapy. Although the therapeutic schedule used in the majority of these patients (interferon alpha monotherapy) is no longer the standard of care, the occurrence of SVR versus persistent replication is a relevant model for the study of virus related versus non virus related steatosis. Steatosis improvement was significantly more frequent in SVR than in NR $(64 \% \vee 31 \%$, respectively; $\mathrm{p}<0.004)$, and this was observed almost exclusively in those infected with genotype 3 where the difference in steatosis improvement between patients who cleared the virus and those who did not was great $(91 \% v 19 \%)$. In addition, multivariate analysis showed that four factors were independently associated with steatosis improvement, including sustained virological response, initial severe steatosis, genotype 3, and BMI. This finding is a very strong argument in favour of direct involvement of HCV in the pathogenesis of hepatic steatosis. It is in keeping with data from Rubbia-Brandt et al who showed that viral clearance was paralleled by the disappearance of steatosis in two patients treated with interferon-alpha, ${ }^{4}$ with another study ${ }^{5}$ reporting an overall decrease in hepatic steatosis in patients achieving a sustained virological response (but given the small number of patients, no genotype specific relationship could be proved), and the recent report of disappearance or reduction in steatosis in 12 selected patients undergoing antiviral therapy who achieved a sustained virological response. $^{18}$ It must be stressed that, in the present study, steatosis evolution after antiviral therapy was assessed in a large series of patients carefully selected among nearly 700 patients followed in our centre with thorough virological characterisation, by a single pathologist, using a validated and reproducible scoring system..$^{21} 22$

Potential confounding variables such as loss of weight or decrease in alcohol intake could account for the improvement in steatosis in SVR infected with genotype 3. However, this hypothesis is unlikely because (a) patients with a BMI $>28 \mathrm{~kg} / \mathrm{m}^{2}$ or excessive alcohol intake prior to treatment initiation were excluded and (b) there was no significant change in BMI or alcohol intake between the pre and posttreatment biopsies among SVR. Moreover, the time elapsed between the end of treatment and the post-treatment liver biopsy, another factor that could influence steatosis changes, did not differ between SVR and NR or between patients infected with genotype 3 and non-3 genotypes.

Although this study provides strong evidence for a role of $\mathrm{HCV}$ genotype 3 in the genesis of steatosis, the mechanisms underlying steatosis in HCV genotype 3 infection remain to be identified. A role for the HCV core protein has been suggested: HCV core protein has been shown to interfere with lipid metabolism in cell cultures. ${ }^{23}$ Two independently derived transgenic mouse lines expressing the HCV core protein ${ }^{13}$ or structural and non-structural proteins ${ }^{14}$ developed progressive hepatic steatosis with mitochondrial dysfunction and production of reactive oxygen species. ${ }^{24}$ Additionally, it has recently been shown in another transgenic murine model that the HCV core protein was able to modify hepatic very low density lipoprotein assembly and secretion by reducing microsomal triglyceride transfer protein activity. ${ }^{25}$ It should be stressed however that in contrast with infected humans, where steatosis is associated with genotype 3, all of these models have used constructs derived from HCV genotype 1 isolates. ${ }^{26}$ Interestingly, in patients with genotype 3 infection, hypobetalipoproteinaemia was more commonly seen than in patients with genotype 1 , and this has been proposed as a possible mechanism for steatosis. ${ }^{5}$ In addition, a significant correlation between the severity of hepatic steatosis and the titre of intrahepatic HCV RNA was found in patients infected with HCV genotype $3 .{ }^{4}$ However, analysis of the nucleocapsid of $14 \mathrm{HCV}$ isolates failed to identify a sequence specifically

\begin{tabular}{|c|c|c|c|}
\hline & OR & $95 \% \mathrm{Cl}$ & $\mathrm{p}$ Value \\
\hline \multicolumn{4}{|l|}{ BMI $\left(\mathrm{kg} / \mathrm{m}^{2}\right)$} \\
\hline$<25$ & 1 & & \\
\hline$>25$ & 0.24 & $0.08-0.73$ & 0.02 \\
\hline \multicolumn{4}{|l|}{ HCV genotype } \\
\hline Non-3 & 1 & & \\
\hline 3 & 2.90 & $0.85-10.0$ & 0.07 \\
\hline \multicolumn{4}{|l|}{ Steatosis grade at time of the first biopsy } \\
\hline Mild ( $<10 \%$ of hepatocytes) & 1 & & \\
\hline Moderate (10-30\% of hepatocytes) & 4.28 & $1.31-14.0$ & 0.02 \\
\hline Severe ( $>30 \%$ of hepatocytes) & 5.50 & $1.54-19.6$ & 0.01 \\
\hline \multicolumn{4}{|l|}{ Response to antiviral therapy } \\
\hline Absence of response & 1 & & \\
\hline Sustained virological response & 6.06 & $1.61-22.9$ & 0.01 \\
\hline
\end{tabular}

BMI, body mass index; HCV, hepatitis $C$ virus; OR, odds ratio. 
associated with the development of steatosis, suggesting that other mechanisms may be involved. ${ }^{4}$

In conclusion, our results showing a significant improvement in steatosis in patients infected with HCV genotype 3 achieving sustained viral clearance provide strong arguments in favour of a direct involvement of HCV in the pathogenesis of liver steatosis. The molecular mechanisms underlying this "cytopathic" effect of HCV remain to be elucidated.

\section{Authors' affiliations}

L Castéra, J-M Pawlotsky, Department of Virology (EA 3489), Centre Hospitalier Universitaire Henri Mondor, Assistance Publique-Hôpitaux de Paris, Université Paris XII, Créteil, France

C Hézode, D Dhumeaux, Department of Virology (EA 3489), and Department of Hepatology and Gastroenterology, Centre Hospitalier Universitaire Henri Mondor, Assistance Publique-Hôpitaux de Paris, Université Paris XII, Créteil, France

F Roudot-Thoraval, Department of Virology (EA 3489), and Department of Public Health, Centre Hospitalier Universitaire Henri Mondor, Assistance Publique-Hôpitaux de Paris, Université Paris XII, Créteil, France

I Lonjon, Department of Hepatology and Gastroenterology, Centre Hospitalier Universitaire Henri Mondor, Assistance Publique-Hôpitaux de Paris, Université Paris XII, Créteil, France

E-S Zafrani, Department of Pathology, Centre Hospitalier Universitaire Henri Mondor, Assistance Publique-Hôpitaux de Paris, Université Paris XII, Créteil, France

\section{REFERENCES}

1 Goodman ZD, Ishak KG. Histopathology of hepatitis $C$ virus infection. Semin Liver Dis 1995;15:70-81.

2 Adinolfi LE, Gambardella M, Andreana A, et al. Steatosis accelerates the progression of liver damage of chronic hepatitis $C$ patients and correlates with specific HCV genotype and visceral obesity. Hepatology 2001;33:1358-64.

3 Hourigan LF, Macdonald GA, Purdie D, et al. Fibrosis in chronic hepatitis C correlates significantly with body mass index and steatosis. Hepatology 1999;29:1215-19.

4 Rubbia-Brandt L, Quadri R, Abid K, et al. Hepatocyte steatosis is a cytopathic effect of hepatitis C virus genotype 3. J Hepatol 2000;33:106-15.

5 Serfaty L, Andreani T, Giral P, et al. Hepatitis C virus induced hypobetalipoproteinemia: a possible mechanism for steatosis in chronic hepatitis C. J Hepatol 2001;34:428-34.

6 Serfaty L, Poujol-Robert A, Carbonell N, et al. Effect of the interaction between steatosis and alcohol intake on liver fibrosis progression in chronic hepatitis $\mathrm{C}$. Am J Gastroenterol 2002;97:1807-12.
7 Castera L, Hezode C, Roudot-thoraval F, et al. Worsening of steatosis is an independent factor of fibrosis progression in untreated patients with chronic hepatitis C and paired liver biopsies. Gut 2003;52:288-92.

8 Czaja AJ, Carpenter HA, Santrach PJ, et al. Host- and disease-specific factors affecting steatosis in chronic hepatitis C. J Hepatol 1998;29:198-206.

9 Mihm S, Fayyazi A, Hartmann H, et al. Analysis of histopathological manifestations of chronic hepatitis $C$ virus infection with respect to virus genotype. Hepatology 1997;25:735-9.

10 Rubbia-Brandt L, Leandro G, Spahr L, et al. Liver steatosis in chronic hepatitis $\mathrm{C}$ : a morphological sign suggesting infection with $\mathrm{HCV}$ genotype 3 . Histopathology 2001;39:119-24.

11 Hui JM, Kench J, Farrell GC, et al. Genotype-specific mechanisms for hepatic steatosis in chronic hepatitis C infection. J Gastroenterol Hepatol 2002:17:873-81.

12 Fujie $\mathrm{H}$, Yotsuyanagi $\mathrm{H}$, Moriya K, et al. Steatosis and intrahepatic hepatitis $\mathrm{C}$ virus in chronic hepatitis. J Med Virol 1999;59:141-5.

13 Moriya K, Yotsuyanagi H, Shintani Y, et al. Hepatitis $\mathrm{C}$ virus core protein induces hepatic steatosis in transgenic mice. J Gen Virol 1997:78:1527-31.

14 Lerat $\mathbf{H}$, Honda $M$, Beard MR, et al. Steatosis and liver cancer in transgenic mice expressing the structural and nonstructural proteins of hepatitis $C$ virus. Gastroenterology 2002;122:352-65.

15 Bellentani S, Tiribelli C. The spectrum of liver disease in the general population: lesson from the Dionysos study. J Hepatol 2001;35:531-7.

16 Monto A, Alonzo J, Watson JJ, et al. Steatosis in chronic hepatitis C: relative contributions of obesity, diabetes mellitus, and alcohol. Hepatology 2002;36:729-36

17 Rubbia-Brandt L, Giostra E, Mentha G, et al. Expression of liver steatosis in hepatitis $C$ virus infection and pattern of response to alpha-interferon. $J$ Hepatol 2001;35:307.

18 Kumar D, Farrell GC, Fung C, et al. Hepatitis C virus genotype 3 is cytopathic to hepatocytes: Reversal of hepatic steatosis after sustained therapeutic response. Hepatology 2002;36:1266-72.

19 Williams GD, Proudfit A, Quinn EA, et al. Variations in quantity-frequency measures of alcohol consumption from a general population survey. Workshop on measurement issues in alcohol consumption. Addiction 1994;89:413-20.

20 Simmonds $\mathbf{P}$, Alberti A, Alter $\mathrm{HJ}$, et al. A proposed system for the nomenclature of hepatitis $C$ viral genotypes. Hepatology 1994;19:1321-4.

21 Intraobserver and interobserver variations in liver biopsy interpretation in patients with chronic hepatitis C. The French METAVIR Cooperative Study Group. Hepatology 1994:20:15-20.

22 Which classification for chronic hepatitis? Lessons from the hepatitis $C$ virus. Groupe METAVIR. Gastroenterol Clin Biol 1994;18:403-6.

23 Barba G, Harper F, Harada T, et al. Hepatitis $C$ virus core protein shows a cytoplasmic localization and associates to cellular lipid storage droplets. Proc Nat Acad Sci USA 1997;94:1200-5.

24 Okuda M, Li K, Beard MR, et al. Mitochondrial injury, oxidative stress, and antioxidant gene expression are induced by hepatitis $C$ virus core protein. Gastroenterology 2002;122:366-75.

25 Perlemuter G, Sabile A, Letteron $P$, et al. Hepatitis $C$ virus core protein inhibits microsomal triglyceride transfer protein activity and very low density lipoprotein secretion: a model of viral-related steatosis. Faseb $J$ 2002; 16:185-94.

26 Negro F. Hepatitis $C$ virus and liver steatosis: Is it the virus? Yes it is, but not always. Hepatology 2002;36:1050-2. 